\title{
Circulating and tumor-based biomarkers predict clinical activity in cancer patients treated with the engineered anti-PD-L1 antibody MPDL3280A
}

\author{
Marcin Kowanetz ${ }^{1 *}$, Christina Rabe², Yuanyuan Xiao ${ }^{1}$, Qun J Wu', Hartmut Koeppen ${ }^{1}$, Cecilia Leddy \\ Rajesh D Patel ${ }^{1}$, John Powderly ${ }^{3}$, Holbrook Kohrt ${ }^{4}$, Scott Gettinger ${ }^{5}$, Jean-Charles Soria ${ }^{6}$, Roy S Herbst ${ }^{7}$, \\ Rupal Desai ${ }^{1}$, Mitchell Denker ${ }^{1}$, Jane Ruppel ${ }^{1}$, Marigold Boe ${ }^{1}$, Rin Nakamura ${ }^{1}$, Ling Fu', Teiko Sumiyoshi ${ }^{1}$, \\ Ahmad Mokatrin ${ }^{1}$, Xiaodong Shen ${ }^{1}$, Gregg Fine ${ }^{1}$, Daniel S Chen ${ }^{1}$, Priti S Hegde \\ From Society for Immunotherapy of Cancer 29th Annual Meeting \\ National Harbor, MD, USA. 6-9 November 2014
}

PD-L1 expressed in the tumor microenvironment regulates Th1 immune responses and mediates cancer immune evasion through interactions with PD-1 or B7.1 receptors on activated T cells. MPDL3280A, an engineered human monoclonal antibody, targets PD-L1 and inhibits its function. To identify immunologic predictive and pharmacodynamic biomarkers of MPDL3280A treatment, we performed a comprehensive analysis of tumors and blood samples collected at baseline and/or on treatment from $\approx 280$ patients with locally advanced or metastatic solid tumors, including NSCLC, RCC, melanoma and bladder cancer. Regardless of tumor type, clinical responses were characterized by PD-L1 expression, the presence of markers of $\mathrm{T}$ cell activation (Th1 gene signature and CTLA4), and the absence of fractalkine at baseline in the tumor microenvironment. Elevated baseline expression of IFN- $\gamma$ and IFN- $\gamma$-inducible genes (e.g., IDO1 and CXCL9) was associated with MPDL3280A response in melanoma but not NSCLC or RCC. On treatment, responding tumors showed increased infiltration of Th1-dominant immune infiltrate and evidence of adaptive PD-L1 up-regulation. In contrast, progressing tumors displayed the following patterns of tumor-infiltrating lymphocytes (TILs) and PD-L1 expression: (1) few/no TILs and absent PD-L1 expression (immunologic ignorance), (2) TILs present with minimal/no PD-L1 expression (non-functional immune responses), or (3) TILs residing solely around the tumor cell mass outer edge (excluded infiltrate), suggesting that resistance to

${ }^{1}$ Genentech, Inc., USA

Full list of author information is available at the end of the article
MPDL3280A may be associated with impaired T cell trafficking and/or function.

Profiling of $\approx 180$ circulating biomarkers revealed that plasma concentrations of IL-18 and interferon-inducible T cell alpha chemoattractant (ITAC) increased in all patients following MPDL3280A treatment, representing a pharmacodynamic measurement of PD-L1 inhibition. In addition, analysis of PBMC showed an increase in T cell activation, as measured by IFN- $\gamma$ and granzymes A and B gene expression in responders following MPDL3280A treatment, consistent with the observations in responding tumors. Baseline soluble PD-L1 was not associated with response. Some indication-specific biomarkers, such as plasma VEGF, decreased in responders with RCC but not with other indications. In NSCLC, a decrease in tumor burden markers, CA-125 and CEA, was associated with response. Similarly, IL-6 and IL-8 were differentially expressed on treatment in responders vs non-responders. Additionally, responders exhibited a decrease in circulating tumor DNA (ctDNA) in plasma, suggesting that ctDNA may be used to monitor MPDL3280A clinical activity in NSCLC.

In conclusion, these data provide general and indicationspecific mechanistic insights into immune checkpoint inhibition, potential mechanisms of response and resistance, as well as identification of potential predictive and pharmacodynamic biomarkers of anti-PD-L1/PD-1 clinical activity across multiple tumor types.

\section{Authors' details}

${ }^{1}$ Genentech, Inc., USA. ${ }^{2}$ Roche Diagnostics GmbH, Germany. ${ }^{3}$ Carolina BioOncology Institute, NC, USA. ${ }^{4}$ Stanford University, Stanford, CA, USA. ${ }^{5}$ Yale 
Cite this article as: Kowanetz et al. Circulating and tumor-based

biomarkers predict clinical activity in cancer patients treated with the

engineered anti-PD-L1 antibody MPDL3280A. Journal for ImmunoTherapy of

Cancer 2014 2(Suppl 3):P136.

Submit your next manuscript to BioMed Central and take full advantage of:

- Convenient online submission

- Thorough peer review

- No space constraints or color figure charges

- Immediate publication on acceptance

- Inclusion in PubMed, CAS, Scopus and Google Scholar

- Research which is freely available for redistribution

Submit your manuscript at www.biomedcentral.com/submit 